Mediterránea Ser. Biol. (1989), n.o 11. Pág. 47-58

\title{
CONTRIBUCIÓN AL CONOCIMIENTO DE LOS NEURÓPTEROS DE CASTELLÓN (INSECTA, NEUROPTEROIDEA, PLANIPENNIA) (1)
}

\author{
por \\ MONSERRAT, V. J. $\left({ }^{*}\right)$ y DÍAZ-ARANDA, L. M. $\left({ }^{* *}\right)$
}

\section{INTRODUCCIÓN}

Continuando con el estudio faunístico de los neurópteros españoles, el objetivo del presente trabajo es dar a conocer la fauna neuropterológica de la provincia de Castellón.

Dicha provincia presenta una extensión de $6.679 \mathrm{Km}^{2}$. Su relieve es accidentado, con altitudes del terreno que oscilan entre el nivel del mar y los 1.183 metros del Peñagolosa en la Sierra de Espadán. La orografía y climatología, típicamente mediterránea, crean un sistema fluvial muy característico, cuya representación más típica son las «ramblas».

En lo que a la vegetación se refiere, las especies arbórèas más representativas son las coníferas, ocupando el pino carrasco (Pinus halepensis Miller) y el pino rodeno (Pinus pinaster Aiton) las cotas comprendidas entre el nivel del mar y los $1.200 \mathrm{~m}$. de altitud. El pino laricio (Pinus nigra Arnold) y el silvestre (Pinus sylvestris L.), mucho menos abundantes, cohabitan en las zonas altas del Maestrazgo, por encima de los $1.000 \mathrm{~m}$. De menor importancia son los enebros (Juniperus oxycedrus L.) y sabinas (Juniperus thurifera L.), que aparecen en manchas aisladas.

Entre las frondosas, menos representadas que las coníferas, la encina (Quercus rotundifolia Lam.) se encuentra repartida por toda la provincia. Existe una

(**) Departamento de Biología Animal. Universidad de Alcalá de Henares. 28871 Alcalá de Henares. Madrid.

$\left({ }^{*}\right)$ Departamento de Biología Animal 1. Facultad de Biología. Universidad Complutense. 28040 Madrid.

El presente trabajo forma parte del proyecto de investigación PB85-0236 de la C.I.C.Y.T.

(1) NOTA: Por problemas de impresión, reproducimos nuevamente este artículo que apareció en Mediterránea n. ${ }^{\circ} 10$ p. 87-94. 
mancha de alcornoque (Quercus suber L.) en la Sierra de Espadán y pequeñas cantidades de quejigo (Quercus faginea Lam.) en las zonas altas del Maestrazgo.

Por último, otras especies de frondosas, aunque menos representativas, que debemos citar son el algarrobo (Ceratonia siliqua L.), la pistacea (Pistacia lentiscus L.), el chopo (Populus pyramidalis Rozan), etc.

\section{MÉTODO}

Para la toma de datos se eligieron 14 localidades de la provincia que pudieran resultar representativas de los distintos biotopos existentes en la misma. Cada localidad fue visitada dos veces en primavera y dos en verano de 1987.

Con el fin de uniformar y en posteriores trabajos poder cuantificar los datos obtenidos durante los muestreos, se estableció una unidad de esfuerzo de 25 «golpes de manga» en cada especie arbórea existente en cada una de las localidades, para poder así recolectar las especies de neurópteros que presentan hábitos nocturnos y pasan el período de insolación entre la vegetación mencionada. Asimismo, para capturar las especies que, aunque poseen actividad nocturna, pasan el período de insolación entre la vegetación herbácea o para aquellas otras que presentan hábitos diurnos, la unidad de esfuerzo complementaria establecida fue de 20 minutos en cada localidad.

Por otro lado, para tratar de recolectar las especies de biología menos generalizada, se utilizó durante la noche trampa de luz negra e incandescente.

Se han colectado ejemplares en todas las localidades elegidas como puntos de muestreo; su altitud y coordenadas UTM se indican a continuación:

1. Alcalá de Chivert, $290 \mathrm{~m}$., 31TBE6465

2. Barracas, $950 \mathrm{~m}$., 30TXK9632

3. Barranco de Surrach, $300 \mathrm{~m} ., 31 \mathrm{TBE} 7588$

4. Benasal, $800 \mathrm{~m} ., 30$ TYK 4373

5. Castillo de Villamalefa, $740 \mathrm{~m} ., 30 \mathrm{TYK} 2345$

6. Cortes de Arenoso, 770 m., 30TYK0951

7. Cuestas de Ragudo, 820 m., 30SXK 9828

8. Monasterio de Vallivana, $600 \mathrm{~m}$., $31 \mathrm{TBE} 4696$

9. Pobleta, La, 1.030 m., 30TYL4612

10. Ribesalbes, 320 m., 30TYK 3233

11. San Miguel de la Puebla, 1.300 m., 30TYK2882

12. Tales, $280 \mathrm{~m}$., 30 SYK 3025

13. Torre Miró, 1.080 m., 30TYL4806

14. Villafranca del Cid, $1.120 \mathrm{~m}$., 30TYK3278

\section{MATERIAL ESTUDIADO}

Se han recolectado durante los muestreos 556 ejemplares pertenecientes a 42 especies de neurópteros, de las que se anotan las referencias de esta provincia existentes en la bibliografía. De dichas especies, 31 han resultado ser nuevas para la fauna de Castellón, indicándose este hecho en el texto mediante un asterisco.

Con el fin de completar los datos obtenidos, se incluyen aquellas especies no colectadas por nosotros y que han sido citadas en la provincia por otros 
autores, incrementándose así a 51 el número de especies recogidas en este trabajo.

Para anotar el material colectado se indican las localidades según la numeración establecida en la lista anterior y se señalan la fecha de recolección, el número de ejemplares machos (m) y hembras (h) y, en su caso, la planta sobre la cual fueron capturados.

El material colectado por los autores queda depositado en la colección del Departamento de Biología Animal de la Universidad de Alcalá de Henares.

PLANIPENNIA (HANDLIRSCH, 1908)

Myrmeleontidae LATREILLE, 1803

Myrmeleon (Myrmeleon) formicarius LINNAEUS, 1767

Especie de distribución paleártica conocida en la provincia por las citas de NAVAS $(1913,1923)$.

Myrmeleon (Morter) hyalinus OLIVIER, 1811

Elemento holomediterráneo del que la única cita existente en la provincia se debe a NAVAS (1922).

Myrmeleon (Morter) inconspicuus RAMBUR, 1842

NAVAS (1922) y VIDAL Y LÓPEZ (1943) citan esta especie, de distribución holomediterránea, en la provincia.

\section{Marcronemurus appendiculatus (LATREILLE, 1807)}

Especie de distribución mediterránea occidental, citada por NAVAS (1921, 1922) y MONSERRAT (1985). 1: 4-VIII-87, 3h. 5: 5-VIII-87, 2m., 1h. 6: 5VIII-87, 7h. 9: 5-VIII-87, 1h. 12: 20-VI-87, $1 \mathrm{~m}$.

Neuroleon nemausiensis (BORKHAUSEN, 1791)

Especie holomediterránea citada como Nelees nemausiensis por NAVAS (1922) en la provincia. 6: 5-VIII-87, 1 h.

Neuroleon ocreatus (NAVAS, 1904)

Especie de distribución mediterránea occidental, conocida en la provincia por las citas de NAVAS (1913) y VIDAL Y LÓPEZ (1943).

Neuroleon arenarius (NAVAS, 1904)

Elemento distribuido por la región holomediterránea, citado en la provincia por NAVAS (1922, 1923) y VIDAL Y LÓPEZ (1943).

Nemoleon notatus (RAMBUR, 1842)

Especie conocida de la región etiópica, extendiéndose al Norte de África, Baleares y Este y Sur de la Península Ibérica. NAVAS (1922) y MONSERRAT (1985) la citan en la provincia. 
Elemento del Mediterráneo occidental, característico de praderas soleadas y pedregosas. 2: 20-VI-87, $2 \mathrm{~m}$. 12: $20-\mathrm{VI}-87,1 \mathrm{~m}$.

Ascalaphidae RAMBUR, 1842

* Libelloides longicornis (LINNAEUS, 1764)

Especie de distribución mediterránea occidental, característica de zonas altas y frescas. 2: 20-VI-87, 2m., 1h. 14: 19-VI-87, 1m., $2 \mathrm{~h}$.

\section{Libelloides baeticus (RAMBUR, 1842)}

Elemento ibérico característico de zonas bajas, donde es frecuente observarlo posado sobre gramíneas. 4: 19-VI-87, 2m. 2: 20-VI-87, 3m., $2 \mathrm{~h}$.

\section{Libelloides cunii (SELYS-LONGCHAMPS, 1880)}

Especie endémica del cuadrante Noreste de la Península Ibérica, citada en la provincia por NAVAS $(1921,1923,1924)$ y VIDAL Y LÓPEZ (1943). AISTLEITNER (1980) la cita como $L$. baeticus cunii. La localidad donde fueron colectados los ejemplares parece estar situada en el área común a los límites de distribución de $L$. baeticus y $L$. cunii, donde ambas especies son simpátridas. 4: 19-VI-87, 4m., 3h.

Bubopsis agrioides (RAMBUR, 1842)

Especie conocida del Mediterráneo occidental, citada en la provincia por MONSERRAT (1985).

\section{Dilaridae NEWMAN, 1853}

* Dilar meridionalis HAGEN, 1886

Elemento de distribución mediterránea occidental del que se han capturado 2 ejemplares, uno a la luz y otro sobre $J$. thurifera. 2: 20-VI-87, $1 \mathrm{~m}$. 9: 18-VI-87, $1 \mathrm{~m}$.

Dilar dissimilis NAVAS, 1903

Especie ibérica cuya cita en la provincia se debe a MONSERRAT (1988).

Hemerobiidae LATREILLE, 1803

* Wesmaelius (Kimminsia) subnebulosus (STEPHENS, 1836)

Distribuida por la región holártica, parece tratarse de una especie euroica, habiéndose colectado 2 ejemplares sobre $J$. thurifera y $P$. sylvestris y 1 en $P$. lentiscus y $Q$. rotundifolia. 2: 29-IV-87, $1 \mathrm{~m}$.; 20-VI-87, $1 \mathrm{~h} .3: 28-\mathrm{IV}-87,1 \mathrm{~h}$. 11: 19-VI-87, 1h.; 4-VIII-87, 1m. 14: 4-VIII-87, 1 h.

\section{Wesmaelius (Kimminsia) navasi (ANDREU, 1911)}

Esta especie, distribuida por las zonas xéricas del límite meridional de la región paleártica occidental, está escasamente citada en nuestra fauna, donde 
presenta una marcada tendencia litoral. 10: 13-IX-87, 2m., sobre C. siliqua. MONSERRAT (1983) cita esta especie en la provincia.

* Sympherobius (Sympherobius) elegans (STEPHENS, 1836)

Especie ampliamente distribuida en el continente europeo, de la que hemos capturado 6 ejemplares sobre $J$. thurifera y 1 en $P$. lentiscus y $C$. siliqua. 2: 20-VI-87, 1m., 1h.; 5-VIII-87, 1m., 3h. 3: 28-IV-87, 1m. 10: 29-IV-87, $1 \mathrm{~h}$.

*Sympherobius (Sympherobius) pygmaeus (RAMBUR, 1842)

Especie holomediterránea. Se ha colectado un único ejemplar, sobre $P o$ pulus pyramidalis. 6: 5-VIII-87, $1 \mathrm{~h}$.

*Sympherobius (Niremberge) fuscescens (WALLENGREN, 1863)

Elemento paleártico, normalmente asociado a pinos y circunscrito a zonas montañosas. 11: 4-VIII-87, $1 \mathrm{~m}$., sobre $P$. sylvestris.

* Hemerobius (Hemerobius) nitidulus FABRICIUS, 1777

Conocido de la región paleártica occidental, asociado a coníferas. Todos los ejemplares fueron colectados sobre $P$. sylvestris. 11: 28-IV-87, 1h.; 19-VI87, 1h.; 4-VIII-87, 1m., 1 h.

* Hemerobius (Hemerobius) stigma STEPHENS, 1836

Esta especie de distribución holártica presenta, al igual que las anteriores, una fuerte tendencia a asociarse a pinos. 10: 20-VI-87, 1h. 11: 19-VI-87, 1m., 1h.; 4-VIII-87, 3h.; 5 de ellos sobre $P$. sylvestris y 1 en trampa de luz.

\section{Chrysopidae SCHNEIDER, 1851}

Italochrysa italica (ROSSI, 1790)

Elemento holomediterráneo, citado por MONSERRAT (1986) en la provincia. VIDAL Y LÓPEZ (1943) cita esta especie dentro del género Nothochry sa. 9: 4-VIII-87, 1h., sobre $P$. halepensis.

Chrysopa septempunctata WESMAEL, 1841

Esta especie, de distribución paleártica, ha sido citada dentro del género Cintameva por VIDAL Y LÓPEZ (1943) en la provincia. 1: 19-VI-87, 1m.; 4-VIII-87, 1h. 3: 28-IV-87, 1h. Todos los ejemplares fueron colectados sobre C. siliqua.

\section{Chrysopa formosa BRAUER, 1850}

Elemento paleártico del que se ha recolectado una hembra en $P$. halepensis. 6: 5-VIII-87. La cita de esta especie en la provincia, dentro del género Cintameva se debe a VIDAL Y LÓPEZ (1943).

\section{* Chrysopa regalis NAVAS, 1915}

Especie de distribución ibérica, muy escasamente citada en nuestra fauna. 5: 19-VI-87, 1h.; 5-VIII-87, $1 \mathrm{~m}$. Ambos sobre $P$. halepensis. 
* Mallada prasinus (BURMEISTER, 1839)

Especie paleártica muy frecuente en la provincia, donde se han colectado $29,9,6$ y 5 ejemplares sobre $Q$. rotundifolia, $C$. siliqua, $J$. oxycedrus y $Q$. faginea, respectivamente; 2 en Pinus halepensis, 2 sobre Populus pyramidalis y 1 en Pinus sylvestris. 1: 28-IV-87, 1m., 1h.; 19-VI-87, 2m., 2h.; 4-VIII-87, 2m. 4: 28-IV-87, 6m., 9h; 19-VI-87, 3m., 2h; 4-VIII-87, 1m., 1h.; 13-IX-87, 1h. 6: 5-VIII-87, 2m. 9: 27-IV-87, 2h.; 18-VI-87, 2m.; 13-IX-87, 2h. 10: 13IX-87, 1h. 11: 4-VIII-87,1h. 13: 27-IV-87, 1h.; 19-VI-87, 2m., 2h.; 4-VIII-87, 1h. 14: 28-IV-87, 1m., 1h.; 4-VIII-87, 2m., 3h.

Mallada venosus (RAMBUR, 1842)

Elemento paleártico occidental, conocido en la provincia por la cita de MONSERRAT (1986).

\section{* Mallada genei (RAMBUR, 1842)}

Especie de distribución holomediterránea, asociada a zonas secas. Ha sido capturado un único ejemplar, sobre Pistacia lentiscus. 3: 4-VIII-87, 1 h.

\section{* Mallada clathratus (SCHNEIDER, 1845)}

Especie distribuida por la región mediterránea septentrional. Escasamente citada y de biología prácticamente desconocida. 11: 4-VIII-87, 1h., en $P$. sylvestris.

* Mallada flavifrons (BRAUER, 1850)

Especie paleártica occidental de la cual se han colectado 18 ejemplares sobre C. siliqua y 1 en Pinus halepensis y Pistacia lentiscus. 1: 28-IV-87, 1m.; 19-VI-87, 2m., 1h.; 4-VIII-87, 1m. 3: 28-IV-87, 1m.; 19-VI-87, 2m., 1h.; 4VIII-87, 1h. 6: 12-IX-87, 1h. 10: 29-IV-87, 5m., 1h.; 20-VI-87, 2m, 1 h.

\section{* Mallada granadensis (PICTET, 1865)}

Elemento del Mediterráneo occidental del que únicamente se ha colectado 1 hembra sobre $P$. halepensis. 5: 19-VI-87, 1 h.

\section{* Mallada picteti (McLACHLAN, 1880)}

Especie de distribución idéntica a la anterior. Bastante frecuente en la provincia, donde parece ocupar las zonas de mayor influencia mediterránea de la misma. 1: 28-IV-87, 5m., 2h.; 19-VI-87, 3m., 4h.; 4-VIII-87, 1h; 11-IX-87, 2h. 3: 28-IV-87, 2m.; 19-VI-87, 2m., 1h.; 4-VIII-87, 1m., 1h. 6: 19-VI-87, 1h.; 5-VIII-87, 1h. 7: 5-VIII-87, 1h. 10: 29-IV-87, 3m., 6h. De entre ellos, 31 ejemplares fueron colectados sobre Ceratonia siliqua, 2 en Pinus halepensis, otros 2 sobre Pistacia lentiscus y 1 en Populus pyramidalis.

\section{* Mallada subcubitalis (NAVAS, 1901)}

Elemento distribuido por la región mediterránea occidental. 2: 20-VI-87, $1 \mathrm{~m}$., sobre $J$. thurifera. 
* Mallada ibericus (NAVAS, 1903)

Especie ibérica de la que se han colectado 13 ejemplares sobre $P$. halepensis, 6 en $J$. thurifera y 4 sobre $C$. siliqua. 1: 28-IV-87, 1m; 19-VI-87, 2m., $1 \mathrm{~h}$. 2: 20-VI-87, 1m., 1h.; 5-VIII-87, 2m., 1h.; 13-IX-87, 1h. 5: 28-IV-87, 5m., 1h.; 19-VI-87, 1m., 4h.; 5-VIII-87, 1m.; 12-IX-87, $1 \mathrm{~h}$.

\section{Chrysoperla carnea (STEPHENS, 1836)}

Especie de distribución prácticamente cosmopolita, cuya cita en la provincia, como Chrysopa vulgaris se debe a VIDAL Y LÓPEZ (1943). 1: 28-IV87, 1m.; 19-VI-87, 2m., 3h. 2: 20-VI-87, 2m., 1h.; 5-VIII-87, 1h. 3: 28-IV-87, 1m., 1h. 4: 19-VI-87, 3m., 2h.; 4-VIII-87, 1m.; 13-IX-87, 1m. 6: 19-VI-87, 3m., 1h.; 12-IX-87, 1m. 9: 27-IV-87, 1h.; 19-VI-87, 3m., 1h.; 4-VIII-87, 1m. 10: 29-IV-87, 1m., 1h. 11: 4-VIII-87, 1m., 1h. 13: 27-IV-87, 1h.; 19-VI-87, 1m., 1h.; 4-VIII-87, 2m. 14: 19-VI-87, 2m., 2h.; 4-VIII-87, 2m., 4h.; 11-IX-87, 2m., $3 \mathrm{~h}$. De entre ellos, 21 fueron colectados sobre $Q$. rotundifolia, 10 en $C$. siliqua, 6 en $Q$. faginea, 5 en $J$. oxycedrus, otros 5 en Populus pyramidalis y 4 , 2 y 1 sobre $J$. thurifera, $P$. sylvestris y $P$. halepensis, respectivamente.

\section{* Chrysoperla mediterranea (HÖLZEL, 1972)}

Elemento mediterráneo occidental, en nuestra fauna está frecuentemente asociado a Pinus halepensis, al que acompaña en su distribución. Hemos colectado 7 ejemplares sobre dicho pino y 1 en $J$. oxycedrus y $J$. thurifera. 2: 13-IX-87, 1h. 7: 13-IX-87, 1h. 9: 27-IV-87, 1m.; 19-VI-87, 3m., 1h.; 10-IX87, 1m. 13: 4-VIII-87, 1h.

Coniopterygidae BURMEISTER, 1839

* Aleuropteryx loewii KLAPALEK, 1894

Especie de distribución paleártica occidental, normalmente asociada a pinos, habiéndose capturado 11 ejemplares sobre $P$. sylvestris y 4 en $P$. halepensis. 5: 28-IV-87, 1m. 6: 19-VI-87, 1h. 9: 18-VI-87, 2h. 11: 19-VI-87, 4m., 6h.; 4-VIII-87, $1 \mathrm{~h}$.

\section{Aleuropteryx juniperi OHM, 1968}

Conocida de la región holártica, esta especie ha sido citada anteriormente en la provincia por OHM (1968). Presenta una fuerte tendencia a asociarse con especies del género Juniperus, donde hemos colectado 1 hembra. 2: 20-VI-87, sobre $J$. thurifera.

\section{* Aleuropteryx iberica MONSERRAT, 1977}

Especie distribuida por la región mediterránea occidental, de la cual siguen colectándose ejemplares sobre Quercus esclerófilos. 4: 19-VI-87, 1h. 9: 18-VI-87, 1m. 14: 19-VI-87, 2m., 1h. Todos ellos sobre $Q$. rotundifolia.

\section{* Helicoconis pseudolutea OHM, 1965}

Se trata de una especie cuya distribución coincide con el límite meridional de la región paleártica occidental y de la que se han colectado 13 ejemplares 
sobre $Q$. rotundifolia y 1 en $Q$. faginea, Pinus halepensis y Populus pyramidalis. 4: 19-VI-87, 2m. 6: 19-VI-87, 1m. 9: 18-VI-87, 1m. 14: 19-VI-87, 1m., 1h.

\section{* Coniopteryx.(Coniopteryx) parthenia (NAVAS \& MARCET, 1910)}

Elemento paleártico asociado a coníferas, habiéndose capturado $49,5,4$ y 3 ejemplares sobre $P$. halepensis, $P$. sylvestris, trampa de luz y $J$. thurifera. 2: 29-IV-87, 1m.; 20-VI-87, 2m. 5: 28-IV-87, 3m., 11h.; 19-VI-87, 5m., 5h.; 12-IX-87, 2m., 3h. 6: 28-IV-87, 1m., 1h.; 19-VI-87, 5m., 2h.; 5-VIII-87, 2h. 7: 29-IV-87, 2m.; 5-VIII-87, 1h. 8: 27-IV-87, 1m., 1h. 9: 27-IV-87, 2m., 4h. 10: 20-VI-87, 1m., 1h. 11: 28-IV-87, 1h.; 19-VI-87, 2h.; 4-VIII-87, 1h.; 11-IX87,1 h.

\section{* Coniopteryx (Coniopteryx) borealis TJEDER, 1930}

Especie de distribución paleártica occidental, de la cual se han colectado 8 ejemplares sobre Pinus halepensis, 3 en Populus pyramidalis y 2 sobre Ceratonia siliqua. 5: 5-VIII-87, 1m., 7h. 6: 5-VIII-87, 1m., 1h.; 12-IX-87, 1m. 10: 13-IX-87, 1m., 1h.

\section{* Coniopteryx (Coniopteryx) ezequi MONSERRAT, 1984}

Elemento ibérico del que se sigue confirmando su asociación con el género Juniperus, habiéndose colectado 21 ejemplares sobre $J$. oxycedrus y 1 en P. halepensis. 2: 29-IV-87, 6m., 1h.; 20-VI-87, 2h.; 5-VIII-87, 2m., 6h. 6: 19VI-87, 1m. 13: 27-IV-87, 2m.; 19-VI-87, 1h.; 4-VIII-87, $1 \mathrm{~h}$.

\section{* Coniopteryx (Holoconiopteryx) haematica McLACHLAN, 1868}

Especie conocida de la región paleártica occidental, abundante y bien representada en la provincia. 1: 19-VI-87, 5m., 5h.; 4-VIII-87, 1m., 1h. 3: 28IV-87, 2m., 5h; 19-VI-87, 1m., 3h.; 4-VIII-87, 2m., 1h. 4: 19-VI-87, 1h. 6: 28-IV-87, 1m. 9: 27-IV-87, 1m., 1h.; 4-VIII-87, 1m. 10: 29-IV-87, 1m., 1h.; 20-VI-87, 4m., 4h. 12: 20-VI-87, 2m., 2h.; 13-IX-87, 2m., 1h. 14: 28-IV-87, 1h.; 19-VI-87, 2h.; 4-VIII-87, 1h. De entre ellos, 40 ejemplares fueron capturados en $C$. siliqua, 7 sobre $Q$. rotundifolia, 3 en Pistacia lentiscus y 1 sobre Pinus halepensis y Populus pyramidalis.

\section{* Coniopteryx (Metaconiopteryx) tjederi KIMMINS, 1934}

Elemento holomediterráneo del cual fueron colectados 5 ejemplares sobre Populus pyramidalis, 3 en $Q$. rotundifolia y 1 sobre $J$. oxycedrus. 4: 28IV-87, 1m., 2h. 6: 5-VIII-87, 2m., 3h. 13: 4-VIII-87, 1m.

\section{* Coniopteryx (Xeroconiopteryx) atlasensis MEINANDER, 1963}

Especie conocida de las zonas térmicas del límite meridional de la región paleártica occidental. Circunscrita a zonas secas, se han colectado todos los ejemplares sobre C. siliqua. 3: 28-IV-87, 2m. 10: 29-IV-87, 1m., 3h. 
* Coniopteryx (Xeroconiopteryx) loipetsederi ASPÖCK, 1963

Especie de distribución mediterránea septentrional, de la que únicamente se ha colectado 1 ejemplar. 2: 29-IV-87, $1 \mathrm{~m}$., sobre $J$. thurifera.

* Semidalis aleyrodiformis (STEPHENS, 1836)

Elemento paleártico, extendido a la región oriental. Hemos capturado 9 , 8, 4 y 3 ejemplares sobre Pinus halepensis, Pistacia lentiscus, $C$. siliqua y $Q$. faginea, respectivamente. 1: 28-IV-87, 1m. 3: 28-IV-87, 6m., 1h.; 4-VIII-87, 1m. 5: 28-IV-87, 4m., 3h.; 20-VI-87, 1h.; 12-IX-87, 1m. 10: 29-IV-87, 1h.; 13IX-87, 2h. 14: 19-VI-87, 1h; 4-VIII-87, $2 \mathrm{~m}$.

* Semidalis pseudouncinata MEINANDER, 1963

Especie distribuida por la región mediterránea occidental, normalmente asociada a enebros y sabinas, donde se han colectado 7 y 2 ejemplares respectivamente. 2: 29-IV-87, 1h.; 5-VIII-87, 1h.; 13: 27-IV-87, 3m.; 19-VI-87, 1m., 3h.

Conwentzia psociformis (CURTIS, 1834)

Elemento holártico, probablemente extendido por acción humana. Esta especie, citada por MONSERRAT (1984) en la provincia, presenta una clara tendencia a asociarse a planifolios. Ha resultado muy abundante en la provincia, donde se han colectado 54 ejemplares sobre $C$. siliqua y 1 en $P$. lentiscus. 1: 28-IV-87, 2h; 19-VI-87, 4m., 4h.; 4-VIII-87, 1h.; 11-IX-87, 1m. 3: 28-IV87, 2m.; 19-VI-87, 3m., 5h. 10: 29-IV-87, 9m., 2h.; 20-VI-87, 6m., 3h. 12: 20VI-87, 5m., $8 \mathrm{~h}$.

\section{COMPOSICIÓN BIOGEOGRÁFICA DE LA FAUNA NEUROPTEROLÓGICA DE CASTELLÓN}

Aunque los datos que poseemos sobre la distribución de numerosas especies son parciales y en ocasiones de difícil adjudicación desde el punto de vista biogeográfico, podemos realizar un somero análisis de los neurópteros de la provincia en estudio en función de los datos anotados.

DISTRIBUCIÓN

COSMOPOLITA

HOLÁRTICA

PALEÁRTICA

PALEÁRTICA OCCIDENTAL

HOLOMEDITERRÁNEA

MEDITERRÁNEA OCCIDENTAL

IBÉRICA

ETIÓPICA EREMIAL
N. ${ }^{\circ}$ DE ESPECIES PORCENTAJE

$1.96 \%$

$7.84 \%$

$13.72 \%$

$19.61 \%$

$17.65 \%$

$25.50 \%$

$43.15 \%$

$11.76 \%$

$1.96 \%$ 
Como era de esperar, dada la ubicación de la provincia, destacan los elementos de distribución mediterránea, siendo más frecuentes los mediterráneo occidentales.

La riqueza de medios existente en la provincia, así como su gradación altitudinal, permite la existencia de otros elementos no exclusivamente mediterráneos, bien holárticos o bien paleárticos, que alcanzan una elevada proporción en su composición faunística.

La influencia del Sistema Ibérico aporta un sustancial número de elementos de carácter endémico que enriquecen su fauna.

Por último, las condiciones xéricas existentes en algunos puntos de la provincia permiten la presencia de algún elemento de carácter eremial digno de destacarse.

\section{DISTRIBUCIÓN DE LAS ESPECIES EN FUNCIÓN DE LA VEGETACIÓN Y EL MEDIO}

Debido a que son numerosas las especies de Planipennia cuya biología es prácticamente desconocida y que muchas de ellas son poco frecuentes o sus poblaciones se encuentran aparentemente localizadas, no es fácil asignarlas a un medio determinado, máxime teniendo en cuenta que, con frecuencia, una misma especie puede aparecer asociada a diferentes fanerófitos en función de su adaptación a las distintas condiciones del medio. Así, Conwentzia psociformis, muy frecuente en los robledales del norte de España, mantiene sus exigencias hídricas asociada a Quercus suber en latitudes más meridionales y la encontramos muy comprometida con Ceratonia siliqua en las zonas más xéricas de influencia mediterránea, sobre la que encuentra suficiente humedad para su normal desarrollo en la provincia estudiada. El mismo ejemplo lo encontramos en Coniopteryx haematica, que es típica de los encinares ibéricos y que en Castellón coloniza otros fanerófitos o nanofanerófitos como C. siliqua y Pistacia lentiscus.

También son frecuentes las especies que tienden a segregarse especializándose y ocupando distintos tipos de fanerófitos, evitando así la competencia de otras especies próximas. Pueden servir como ejemplos Chrysoperla mediterranea, que la encontramos casi exclusivamente sobre Pinus halepensis a diferencia de C. carnea, totalmente ubiquista, que aparece sobre cualquier sustrato vegetal, o de Semidalis pseudouncinata, que, especialmente asociada a enebros, evita la presión de $S$. aleyrodiformis, aparentemente ubiquista, o de Wesmaelius navasi, que coloniza medios xéricos, evitando la presencia de $W$. subnebulosus, que posee una biología más generalizada.

En función de los datos conocidos y de los obtenidos en los muestreos realizados, podemos anotar los siguientes comentarios:

Las zonas costeras, ramblas y eriales están ocupados principalmente por Mirmeleónidos, donde encuentran condiciones adecuadas para su desarrollo larvario, así como por otras especies de tendencias xéricas (Bubopsis agrioides, $W$. navasi, etc.).

Las praderas son ocupadas principalmente por Ascaláfidos, buenos voladores que se segregan altitudinalmente, ocupando Libelloides longicornis las cotas más elevadas y $L$. baeticus y $L$. cunii las más bajas. 
Las formaciones vegetales de frondosas, mantienen poblaciones características de Sympherobius pygmaeus, S. elegans, Chrysopa formosa, Coniopteryx haematica o Conwentzia psociformis, restringiéndose en algunas ocasiones a un determinado fanerófito, así Aleuropteryx iberica siempre la encontramos sobre Quercus rotundifolia.

Algunas poblaciones de neurópteros presentan una gran afinidad con las formaciones vegetales aciculifolias, tal es el caso de Mallada ibericus. Otras poseen una mayor especialización, ocupando preferente o exclusivamente especies del género Pinus (Sympherobius fuscescens, , Hemerobius nitidulus, $H$. stigma, Chrysoperla mediterranea, Chrysopa regalis, Aleuropteryx loewii, Coniopteryx parthenia) o especies de Juniperus (Aleuropteryx juniperi, Coniopteryx ezequi, Semidalis pseudouncinata).

Por último, otras especies en la provincia colonizan indistintamente cualquier medio o sustrato vegetal (Chrysoperla carnea, Mallada flavifrons, Semidalis aleyrodiformis, Wesmaelius subnebulosus).

\section{BIBLIOGRAFIA}

AISTLEITNER, E., 1980. Die Arten des Genus Libelloides Tjd. der Iberischen Halbinsel. Entomofauna 1, 14: 234-297.

MONSERRAT, V. J., 1983. Sobre los neurópteros de las Islas Canarias IV: Wesmaelius (Kimminsia) navasi (Andreu, 1911). (Neur., Plan., Hemerobiidae). «Boletín Asoc. Esp. Entom». Vol. 6 (Fasc. 2): 209-224.

- 1984. Nuevos datos sobre los coniopterígidos (Neur., Plan., Coniopterygidae) ibéricos. «Boletín Asoc. Esp. Entom». Vol. 8: 25-34.

- 1985. Nuevos datos sobre los Myrmeleontoidea ibéricos (Neur., Plan., Myrmeleonidae, Ascalaphidae, Nemopteridae). «Bolm. Soc. Port. Ent.»Vol. 2 (suplemento 1): 475-489.

- 1986. Sobre los neurópteros ibéricos (IV) (Neur.). «Boletín Asoc. Esp. Entom». Vol. 10: 95-105.

- 1988. Revisión de los diláridos ibéricos. (Neuropteroidea, Planipennia, Dilaridae). Eos. 64: 175-205.

NAVAS, L., 1913. Myrmeleónidos (Ins., Neur.) de la fauna ibérica. «Congr. Asoc. Esp. Progr. Ciencias». Madrid: 5-37.

- 1921. Mis excursiones científicas del verano de 1919. «Mem. R. Ac. Ci. Art. Barcelona». Vol XVII, n. ${ }^{\circ}$ 6: 143-169.

- 1922. Algunos insectos recogidos por D. Fermín Cervera en las provincias de Tarragona, Castellón y Valencia. «Bol. Soc. Ent. Esp». V: 147-148.

- 1923. Entomología de Catalunya. Neuròpters. Inst. d'Estudis Catalans: Sec. Ciencies (1924): $1-270$.

- 1924. Sinopsis de los neurópteros (Ins.) de la Península Ibérica. Mem. Soc. Ibérica. Cienc. Nat. 4: 1-150.

OHM, P., 1968. Vorläufige Beschreibung einer neuen europäischen Aleuropteryxart (Neuroptera, Coniopterygidae). Ent. Nachrbl. (Wien) 15: 12-15.

VIDAL Y LÓPEZ, M., 1943. Catálogo provisional de los neurópteros de la región levantina. Graellsa» 1 (6): 13-24. 